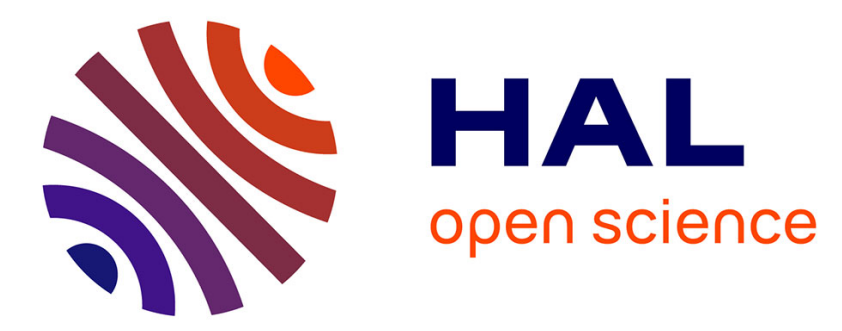

\title{
Sur la radioactivité des gaz qui proviennent de l'eau des sources thermales
}

\author{
Pierre Curie, A. Laborde
}

\section{To cite this version:}

Pierre Curie, A. Laborde. Sur la radioactivité des gaz qui proviennent de l'eau des sources thermales. Radium (Paris), 1906, 3 (7), pp.195-197. 10.1051/radium:0190600307019501 • jpa-00242185

\section{HAL Id: jpa-00242185 https://hal.science/jpa-00242185}

Submitted on 1 Jan 1906

HAL is a multi-disciplinary open access archive for the deposit and dissemination of scientific research documents, whether they are published or not. The documents may come from teaching and research institutions in France or abroad, or from public or private research centers.
L'archive ouverte pluridisciplinaire $\mathbf{H A L}$, est destinée au dépôt et à la diffusion de documents scientifiques de niveau recherche, publiés ou non, émanant des établissements d'enseignement et de recherche français ou étrangers, des laboratoires publics ou privés. 


\title{
Sur la radioactivité des gaz qui proviennent de l'eau des sources thermales
}

\author{
Par P. CURIE et A. LABORDE.
}

$\mathrm{D}$ Ans une publication antérieure ${ }^{2}$, nous avions indiqué quelques sources naturelles d'où se dégageaient spontanément des gaz radioactils; et nous avions classé ces sources d'après leur radio-

1. Xote presentée à l'Aeadémie des Sciences le 11 juin 1906. 2. comples rendus, t. CXXXYIII, 1.1150. activité qui avait été déterminée quantitativement.

Nous avions étudié de nouvelles sources thermales, et, pour quelques-unes de celles dont les gaz dégagés spontanément se sont montrés le plus radioactif's, nous avons recherché la radioactivité de l'eau recueillie an griffon de la source. 
La radioaciivité des gaz a été détcrminéc par la méthode de mesure électrique décrite antérieurement ${ }^{1}$.

Pour extraire des eaux l'émanation radioaclive qu'elles renfermaient en dissolution, nous arons fait bouillir ces eaux dans un ballon de 5 litres muni d'un réfrigérant ascendant, de telle facon que les gaz chassés par ébullition pussent ètre recueillis sur le mercure; quand l'eau étudiéc était fortement chargée d'acide carbonique, nous empèchions ce gaz de se dégager en plıçant de la potasse dans le ballon.

Nous avons laissí bouillir ainsi les eaux pendant une heure environ, et, à plusicurs reprises au cours d'une opération, nous arons fait passer dans le ballon un courant d'air non radioactif qui avait pour but d'entrainer par barbotage les dernières traces d'émation qui pouvaient subsister dans le liquide ou dans l'espace libre des tubes de dégagement. Les gaz ainsi recueillis ont été introduits dans un condensateur cylindrique à anneau de garde et leur radioactivité a été mesurée par la míthodedu quartz piézo-électrique.

Nous arons pu dresser ainsi un tableau dans lequel figurent les résultats des anciennes et des nouvelles qui définissent la quantití d'énanation contenue dans les gaz ou dans les eaux étudiés : cette quantití d'émanation est facile à connaitre quand le condensateur cylindrique utilisé a été étalonné une fois pour toutes avec de l'émanation du radium'.

Cet étalonnage a étí effectué récemment dans de très bonnes conditions par Mme Curie au cours d'un travail qui n'est pas encore publié : les résultats obtenus par Mine Curie nous ont conduits à modilier les nombres fournis à ce sujet dans notre première communication, car ces nombres avaient été déterminés à la suite d'expériences faites avec des solutions de bromure de radium dont le titrage était à notre insu, entaché d'erreur.

D'après Mme Curic, 1 gramme de bromure de radium pur dégage en 1 heửre une quantité d'émanation capable de provoquer, dans un condensateur cylindrique de $1500^{\mathrm{ms}}$ (longueur du condensateur : $12 \mathrm{~cm}, 65$; diamètre du cylindre extérieur : $6^{\mathrm{cm}, 8}$ diamètre de la tige intéricure: $\left.0{ }^{\mathrm{cm}}, 28\right)$, un courant de saturation maximum (3 heures après l'introduction de l'émanation dans le condensateur) de 1,21.10

\begin{tabular}{|c|c|c|c|c|c|}
\hline \multirow{2}{*}{ NOY DE LI SOLRCE } & D.TTE & \multicolumn{2}{|c|}{$\mathrm{G} \cdot \mathrm{A} Z$} & \multicolumn{2}{|c|}{ E.UUX } \\
\hline & L'IXTRACIIJN & $i .10^{3}$ & $n$ & $i_{1} \cdot 10^{i}$ & $n_{1}$ \\
\hline 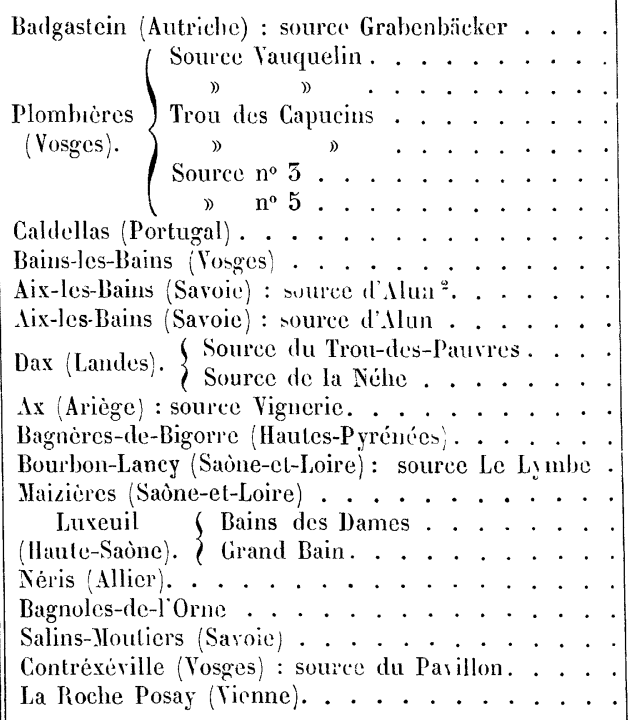 & $\begin{array}{r}4 / 01 \\
1 / 04 \\
3 / 05 \\
3 / 04 \\
8 / 05 \\
1 / 04 \\
1 / 04 \\
4 / 05 \\
3 / 04 \\
2 / 05 \\
8 / 05 \\
11 / 04 \\
11 / 04 \\
10 / 05 \\
10 / 03 \\
1 / 05 \\
12 / 04 \\
2 / 04 \\
2 / 04 \\
5 / 04 \\
2 / 04 \\
1 / 04 \\
2 / 05 \\
3 / 05\end{array}$ & $\begin{array}{c}560 \\
47 \\
52 \\
21 \\
\text { ” } \\
29 \\
28 \\
17 \\
16 \\
16 \\
15 \\
13,3 \\
2,6 \\
10,6 \\
10,6 \\
9,3 \\
6,78 \\
5,70 \\
2,3 \\
4,2 \\
3,3 \\
5 \\
» \\
»\end{array}$ & $\begin{array}{c}39,6 \\
5,17 \\
5,72 \\
2,51 \\
7 \\
5,19 \\
5,08 \\
1.82 \\
1,76 \\
1,76 \\
1,43 \\
1,46 \\
0,28 \\
1,16 \\
1,16 \\
1,03 \\
0,74 \\
0,62 \\
0,25 \\
0,46 \\
0,33 \\
0,35 \\
\searrow \\
》\end{array}$ & 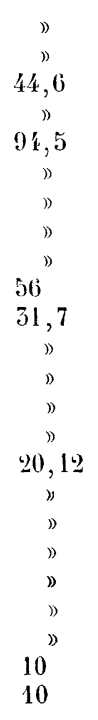 & 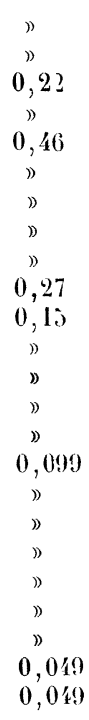 \\
\hline
\end{tabular}

déterminations. Comme précédenment nous avons indiqué dans ce tableau les valeurs du courant $\left(i .10^{5}\right.$ en unités électrostatiques) que l'on obtient dans un condensateur cylindrique déterminé 4 jours après que le gaz étudić a été recueilli à la source; nous avons igalement fait figurer dans ce tableau des nombres

1. Loc. cit.

2. La radioactiviti des sources d'Lix-les-Bains a été observée pour la première fois par II. G.-A Blanc (Phil. Mag., janvier $190 \mathrm{~s})$. unités électrostatiques. Ces mesures ont été faites à $15^{0} \mathrm{C}$., et à la pression atmosphérique normale.

A l'aide de ces données, nous arons pu calculer que, dans nos appareils, un courant de saturation de 1 unité électrostatique est produit par la quantité d'émanation que dégage 1 milligramme de bromure de radium pur en 4,95 minutes, ce courant étant

1. Loc. cit. 
mesuré quand l'émanation a alteint son état d'équilibre de régime avec la radioactivité induite qu'elle crée.

Jans le tableau ci-dessus nous avons fait figuree:

A la colonne 1 : la date de l'extraction (mois et année); A la colonne 2 : le courant de saturation $i .10^{5}$ produit par $450^{\mathrm{cms}}$ de gaz dans un condensateur cylindrique de $450^{\mathrm{ms} s}$, 4 jours après l'extraction, à $15^{\circ} \mathrm{C}$ et à la pression atmosphérique normale;

A la colonne 3 : le nombre $(n)$ de minutes pendant lequel il faudrait laisser séjourner 1 milligramme de bromure de radium pur dans 10 litres d'air pour obtenir le mème courant dans notre appareil qu'avec les gaz étudiés;

A la colonne 4 : le courant $i_{1} \times 10^{5}$ produit dans notre appareil par l'émanation extraite de 10 litres d'eau ầée de 4 jours:

A la colonne 5 : la quantité d'émanation présente dans 10 litres d'eau àgée de 4 jours, cetle quantité d'émanation étant exprimée comme dans le cas de la colonne 5 par le temps $\left(n_{1}\right)$ pendant lequel 1 milligramme de bromure de radium pur produirait cette émanation.

Cauterets (Hautes-Pyrénées) : sources César, dés (Eufs; Le Bois, La Raillière; Eaux-Chaudes (Basses-Prrénées); Eaux-Bonnes (Basses-Pyrénées); Mont-Dore (Puy-de-Dòme); source Bardon, Madeleine; Lamalou (llérault); Rosat (Puy-
de-Dòme); Ogen (Puy-de-Dòme); Source intermittente (Allier); Larderello (Italie) $=$ gaz dont la radioactivité correspond à $i .10^{3}<5$.

Alet (Aude); Châtel-Guyon (Puy-de-Dòme); Montbrunles-Bains; Pougues Saint-Léger (Nièvre); Vichy (Allier) : sources Boussange, Célestins, Lucas, Ilòpital, Grande Grille, Chomel; Forges-les-Eaux (Seine-Inférieure); Saint-Honoréles-Bains (Nièvre); Spa (Belgique) =gaz dont la radioactivité correspond à $i .10^{5}<1$.

Vichy (Allier) : sources Chomel, (irande Grille; Villel (Vosges); Evian (Ilaute-Savoie) : source Cachat =ean $x$ dont la radioactivité correspond à $i_{1} \cdot 10^{5}<5$.

Les nombres qui figurent aux colonnes 4 et 5 se rapportent tous deux à des eaux qui contiennent de l'émanation du radium au moment de leur extraction," mais qui ne contiennent pas de sel de radium en dissolution; en effet, nous avons constaté qu'après avoir conservé ces eaux en vase clos pendant plus de un mois, nous ne pouvions plus en extraire d'émanation radioactive.

Comme nous avous indiqué la radioactivité des gaz et des eaux 4 jours après leur extraction, on peut admettre qu'au griffon de la source elle aurait été deux fois plus forte.

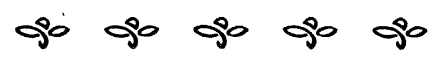

